\title{
Plasmonic magneto-optical nested 2D nanostructures: tailoring responses through effective refractive index
}

\author{
Alexey Shaimanov ${ }^{1,2, *}$ and Alexander Baryshev ${ }^{1,3}$ \\ ${ }^{1}$ All-Russia Research Institute of Automatics, Moscow 127055, Russia \\ ${ }^{2}$ Moscow State University, Moscow 119991, Russia \\ ${ }^{3}$ Moscow Institute of Physics and Technology, Dolgoprudny 141700, Russia
}

\begin{abstract}
Spectra of a nested square two-dimensional lattice of metal nanospheres encased in a magneto-optical host were studied. We show that the magneto-optical response of the nanostructures considerably increases due to the plasmon resonances. Moreover, the optical and magneto-optical responses can be strongly altered with a negligible change in structural parameters.
\end{abstract}

Nanophotonics encompasses studies on artificial nanostructures that support various optical resonances [1]: surface propagating plasmon resonance (SPR) and localized surface plasmon resonances (LSPR). These plasmonic resonances in various magneto-optical (MO) plasmonic systems are useful for the control of light intensity or polarization. The enhancement of polarization rotation is observed in the polarization rotation spectra of plasmonic MO nanostructures and should be understood as a reciprocal phenomenon [2], however, can be useful for a tunable waveplate [3].

To demonstrate a new view on plasmonic MO nanostructures, we introduce a nested square two-dimensional (2D) lattice of gold $(\mathrm{Au})$ nanospheres encased in a magnetic host (a layer bismuth-substituted yttrium iron garnet, Bi:YIG). We analyze spectra of 2D AuBi:YIG nanostructures with square lattices having different periods and discuss LSPRs on single nanospheres and their ensemble. Then, we select a 2D lattice and insert additional nanospheres with radius $\mathbf{R}$, composing another square lattice, into it, thus forming a nested 2D lattice. We will show that the number and spectral positions of the LSPR bands in spectra of the nested Au-Bi:YIG nanostructure are governed by the radius of nanospheres, the MO response can be enhanced at frequencies of LSPRs and it becomes extremely sensitive to the radius of the nanospheres. Two main features are in spectra of such strustures: LSPR (D) band is defined by the lattice period $\mathbf{D}$ and has a large spectral shift as D changes and LSPR on single spherical nanoparticle in the short-wavelength (SW) band at approximately $600 \mathrm{~nm}$ (see Fig. 1(a)).

We compare MO responces of the Au-Bi:YIG nanostructures with square and nested lattices and find interesting features. Unexpectedly, the sign of the angle of polarization

\footnotetext{
* Corresponding author: shaymanov.aleksey@physics.msu.ru
} 
rotation $\theta(\lambda)$ oscillates in the vicinity of LSPR $\left(\mathbf{D}_{\mathbf{1}}\right)$ and is same as that of Bi:YIG at LSPR ( $\left.\mathbf{D}_{2}\right)$ band. Moreover, for the SW range, a negligible change in $\mathbf{R}$ results in the drastic flip-over of the MO response at $\lambda=630 \mathrm{~nm}$ [see the left graph in Fig. 1(b), (c)]. Another interesting feature in the spectra is that the presence of the additional scatterers having the coordinates of $\left(i \mathbf{D}_{\mathbf{3}} x, j \mathbf{D}_{\mathbf{3}} y\right)$ strongly influences the spectral position of LSPR $\left(\mathbf{D}_{1}\right)$. A small change in $\mathbf{R}$ results in the following spectral shifts: $\Delta \lambda\left(\operatorname{LSPR}\left(\mathbf{D}_{1}\right)\right) \approx 5$ $\mathrm{nm}$ and $\left.\Delta \lambda\left(\operatorname{LSPR}\left(\mathbf{D}_{2}\right)\right)\right) \approx 1 \mathrm{~nm}$ at $\Delta \mathbf{R}=1 \mathrm{~nm}$. The observed peculiarities, result from the out-of-phase field oscillation on the neighbor nanoparticles in the case of LSPR $\left(\mathbf{D}_{1}\right)$. In contrast, there is an in-phase dipole-to-dipole interaction of the neighbor nanoparticles in the case of LSPR $\left(\mathbf{D}_{2}\right)$-the likely reason why the MO response does not change sign. It is worth noting that in the vicinity of strong polarization rotation at $635 \mathrm{~nm}$ the light emerging from the Au-BiYIG layer is elliptically polarized, see Fig. 1 (b) and (c). As for polarization states of the waves at LSPR $\left(\mathbf{D}_{2}\right)$ and LSPR $\left(\mathbf{D}_{1}\right)$, they are linear $(\lambda=733 \mathrm{~nm})$ and elliptical $(\lambda \approx 925 \mathrm{~nm})$ polarizations correspondingly, see Fig. 1 (c).
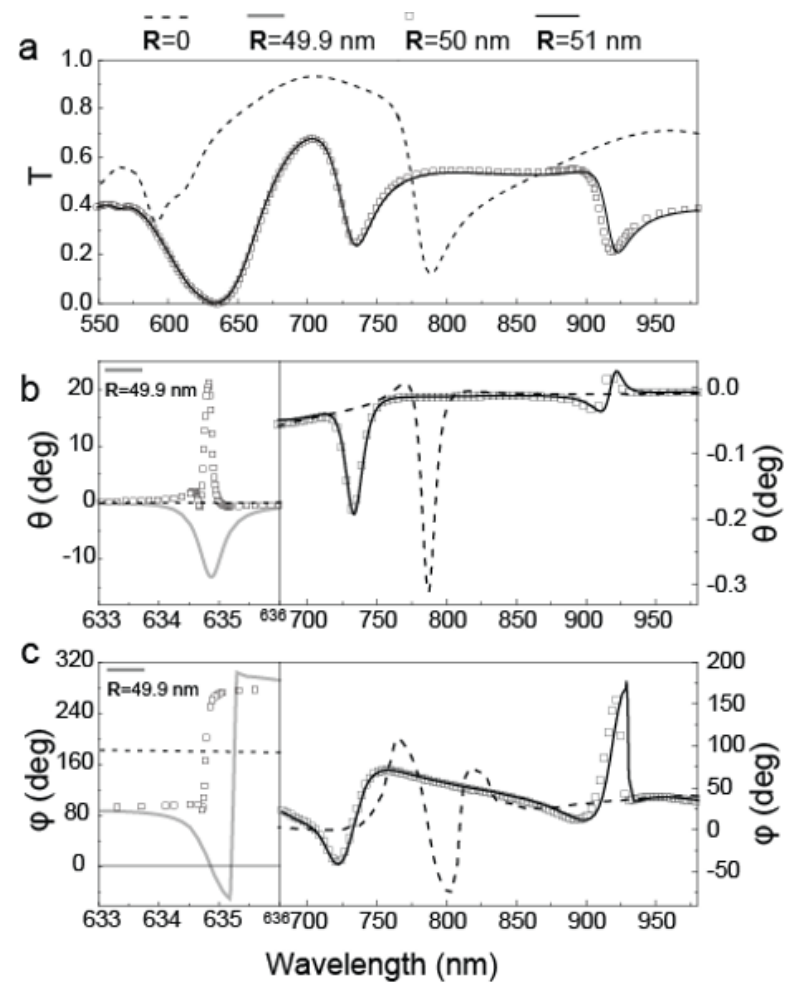

Fig. 1. a) Calculated transmittance (b) polarization rotation spectra and (b) phase shift between the $\mathbf{E}_{x}$ and $\mathbf{E}_{y}$ components for a nested 2D structure with $\mathbf{D}_{\mathbf{1}}=350 \mathrm{~nm}$. The pairs of spectra demonstrate their dependence on a negligible change of $\mathbf{R}$. Spectra of the square $2 \mathrm{D}$ structure with $\mathbf{D}_{\mathbf{1}}=350 \mathrm{~nm}$ are shown for comparison.

\section{References}

1. S. A Maier, Plasmonics: Fundamentals and Applications (Springer, USA, 2007)

2. A. V. Baryshev, H. Uchida, and M. Inoue, J. Opt. Soc. Am. B 30, 2371 (2013)

3. A. V. Baryshev, A. M. Merzlikin, J. Opt. Soc. Am. B 33, 1399 (2016) 